\title{
Estructura poblacional y relaciones ambientales del árbol tropical Nectandra rudis (Lauraceae), una especie rara en el occidente de México
}

\author{
Ramón Cuevas Guzmán ${ }^{1}$, Edmundo García Moya $^{2}$, J. Antonio Vázquez García ${ }^{3}$ \\ \& Nora M. Núñez López ${ }^{1}$ \\ 1 Departamento de Ecología y Recursos Naturales, Centro Universitario de la Costa Sur, 48900 Autlán de Navarro, \\ Jalisco, México; rcuevas@cucsur.udg.mx; nnunez@cucsur.udg.mx \\ 2 Postgrado en Botánica, Campus Montecillo, Colegio de Postgraduados, Montecillo, Estado de México, México; \\ edmundo@colpos.mx \\ 3 Departamento de Botánica y Zoología, Centro Universitario de Ciencias Biológicas y Agropecuarias, Zapopan, \\ Jalisco, México; jvazquez@cucba.udg.mx
}

Recibido 05-IX-2006. Corregido 14-III-2007. Aceptado 14-V-2007.

\begin{abstract}
Population structure and environmental relationships of the tropical tree Nectandra rudis (Lauraceae), a rare species in western Mexico. The tree $N$. rudis is a rare species from western Mexico of which community and population features are unknown. We studied a population in an altitudinal gradient, from 550-1 $850 \mathrm{~m}$ above sea level in the Sierra de Manantlan, Jalisco, Mexico. We established four 60x48 m sample sites at vertical distances of $100 \mathrm{~m}$ along this altitudinal gradient. Within each plot, ten $100 \mathrm{~m}^{2}$ circular sub-sampling units were randomly located. At each unit, we recorded diameter at breast height (dbh) and tree height for all woody vegetation $\geq 2.5 \mathrm{~cm}$ dbh. Basal area, tree density, frequency, species richness and importance values per species and plot. We estimated the vertical structure (total tree height) and diameter( as $\mathrm{M}=5 \log _{10} \mathrm{~N}$ ) for all N. rudis individuals. A direct ordination through Canonical Correspondence Analysis was done, involving amongst other species, edaphic and environmental data matrices. The record of $44 \mathrm{~N}$. rudis individuals, in seven out the 56 plots sampled, represents the most septentrional record for the species and the first in Western Mexico. Its density and basal area represented $4.5 \%$ and $8.7 \%$ respectively of the total estimated for the community. The greatest importance values were observed at $1650 \mathrm{~m}$ above sea level. The population structure of $N$. rudis is structured into five diameter categories in an inverse " $\mathrm{J}$ " shaped distribution. This is a typical behavior observed to occur in the Lauraceae, which produces big seeds of short viability that germinate when there is high soil moisture content. The species tend to form dense seedling banks although only a reduced number of them are able to survive. Species richness varies from 27 to 39 at plot level; the greatest importance values for the plots on which $N$. rudis was found, corresponds to Urera verrucosa (Liebm.) V.W. Steinm., N. rudis, Ficus sp., Beilschmiedia manantlanensis Cuevas y Cochrane, amongst others. Canonical Correspondence Analysis suggests that environmental variables such as rooted trees, crown cover, litter depth and soluble magnesium are the more significant explanatory variables for the distribution and abundance of N. rudis. Rev. Biol. Trop. 56 (1): 247-256. Epub 2008 March 31.
\end{abstract}

Key words: Canonical Correspondence Analysis, Nectandra rudis, populational structure, Sierra de Manantlán, western Mexico.

Nectandra es un género Neotropical y el segundo de la familia Lauraceae con más entidades específicas en América (114), de las cuales treinta se han descrito recientemente (Rohwer 1993). Aunque la mayoría de las especies se encuentran en el trópico americano, algunas rebasan los límites de esta región, como $N$. coriaria (Sw.) Griseb. y N. salicifolia (Humb., Bonpl. y Kunth) Nees e incursionan más hacia el norte. En México se han registrado 19 entidades del género, la mayoría de ellas concentradas en las regiones húmedas o montañas lluviosas de ambas vertientes, desde el nivel del mar hasta cerca de los $3000 \mathrm{~m}$ de altitud (Lorea 2002). 
El registro de Nectandra rudis en el occidente de México, en la Sierra de Manantlán, representa el registro más al norte para el taxon. Previo a este hallazgo, se consideraba un elemento endémico del sur de México y Centroamérica, ya que su distribución registrada era Chiapas, Guatemala y El Salvador, desde los 1600 hasta los $2500 \mathrm{~m}$ de altitud, en bosque mesófilo de montaña. Ante la disyunción geográfica, la poca información sobre las poblaciones de $N$. rudis y las comunidades donde crece, $\mathrm{y}$ ante las necesidades y prioridades de conservación y/o manejo de especies raras de la Reserva de la Biosfera Sierra de Manantlán, se considera pertinente y relevante describir la estructura poblacional y las características estructurales de las comunidades vegetales donde se desarrolla la especie, así como identificar los factores ambientales que podrían estar relacionados con su distribución y abundancia.

\section{MATERIALES Y MÉTODOS}

Área de estudio: los sitios donde se encontró y levantaron censos poblacionales de $N$. rudis, se localizan en el occidente de México, al oeste de la Sierra de Manantlan, en el municipio de Casimiro Castillo, Jalisco, dentro del ejido de Barranca de La Naranjera, el cual cuenta con un área de aproximadamente 9800 ha (Cuevas 2002). La hidrología forma parte de la cuenca del Río Purificación y de la subcuenca El Tecolote. El clima es cálido con dos subgrupos de cálido subhúmedo, $\mathrm{Aw}_{1}(\mathrm{w})$ y $\mathrm{Aw}_{2}$ (w) (Martínez et al. 1991). La precipitación media anual es de $1635 \mathrm{~mm}$, mientras que la temperatura promedio varía entre $\operatorname{los} 25-30{ }^{\circ} \mathrm{C}$, con una mínima de $10{ }^{\circ} \mathrm{C}$ en invierno y una máxima hasta de $44{ }^{\circ} \mathrm{C}$ en primavera (Orozco 2001). El tipo de suelo de mayor extensión en el ejido corresponde a los Regosoles, con áreas cubiertas por Cambisoles y Feozem (Vázquez et al. 1995). Se presentan cuatro tipos de vegetación: bosque tropical caducifolio, bosque tropical subcaducifolio, bosque mesófilo de montaña y bosque de Quercus (Rzedowski 1978, Vázquez et al. 1995). Las poblaciones de $N$. rudis se localizaron en una cañada que corre al este de Casimiro Castillo y finaliza en el rancho de Corralitos; en su parte más alta es conocida como cañada del Alentrisco y en la parte media y baja como barranca o arroyo El Tecolote.

Identificación y distribución del taxon: la especie fue identificada mediante la revisión de la literatura pertinente del género (Rohwer 1993) y corroborada por el Dr. Francisco Lorea Hernández, especialista en las lauráceas de México con sede en el herbario XAL. La distribución geográfica se obtuvo mediante la revisión de ejemplares de los herbarios CHAPA, MEXU, IBUG y ZEA, y se complemento con literatura especializada.

Muestreo y análisis de los datos: la estructura poblacional y de la comunidad se obtuvo a través de un estudio a lo largo de un gradiente altitudinal entre los 550 y los $1850 \mathrm{~m}$. Cuatro sitios de muestreo fueron establecidos a cada $100 \mathrm{~m}$ a través del gradiente altitudinal, y en total 56 en el gradiente. Las parcelas consistieron de un rectángulo de 60x48 m, el cual se dividió en 20 subparcelas de 12x12 m (Vázquez y Givnish 1998, Cuevas et al. 2002), de las cuales 10 fueron seleccionadas aleatoriamente. Dentro de cada una de ellas y partiendo de su centro, se trazó un círculo de radio $=5.64 \mathrm{~m}$ para obtener un área de $100 \mathrm{~m}^{2}$ y $1000 \mathrm{~m}^{2}$ para toda la parcela. En cada círculo de $100 \mathrm{~m}^{2}$ se identificó, registró y midió el diámetro normal y la altura de todas las especies leñosas con diámetro $\geq 2.5 \mathrm{~cm}$. Para cada parcela se obtuvo el área basal, la densidad, la frecuencia y el valor de importancia por especie. El valor de importancia fue calculado como el promedio de la suma de los valores relativos de dominancia, frecuencia y densidad (Curtis y McIntosh 1951).

La estructura vertical de la población se obtuvo midiendo las alturas de todos los individuos con diámetro $\geq 2.5 \mathrm{~cm}$. La estructura diamétrica poblacional de $N$. rudis se basó en todos los individuos encontrados en las parcelas en las que se realizó el muestreo. La obtención 
del número de clases diamétricas se hizo con base en $\mathrm{M}=5 \log _{10} \mathrm{~N}$, donde $\mathrm{M}=$ número de clases diamétricas, $\log _{10}$ es el logaritmo base $10 \mathrm{y}$ $\mathrm{N}=$ número de individuos $\mathrm{y}$, para los intervalos de clase se uso: I.C.=amplitud diamétrica/M.

La caracterización de la estructura de la comunidad donde se encuentra $N$. rudis, se realizó considerando la riqueza de especies, así como la altura y diámetro de todas las especies presentes en las parcelas.

Se consideraron 42 variables edáficas y ambientales en un análisis de selección de variables a través del método "forward" disponible en el programa CANOCO (ter Braak y Smilauer 1998), de las cuales el programa seleccionó en forma automática las variables edáficas: materia orgánica, porcentaje de arena, capacidad de intercambio catiónico, potasio intercambiable, magnesio soluble y bióxido de carbono soluble. También se incluyeron en otra matriz las variables ambientales de altitud, profundidad del horizonte superficial, incidencia de incendios, incidencia de pastoreo, cantidad de árboles caídos y presencia de tocones. Por las pocas parcelas en las cuales se encontró $N$. rudis y debido a las restricciones del programa de análisis multivariable, de no poder analizar matrices con más variables ambientales que unidades de muestreo, fue necesario correr las variables edáficas y las variables ambientales por separado, a través de un Análisis de Correspondencia Canónica (ACC), con el propósito de detectar aquellas que podrían estar asociados con la distribución y abundancia de $N$. rudis. La intensidad de pastoreo y la incidencia de incendios se registraron con base en Olvera et al. (1996). El análisis multivariable se realizó con el programa PC-ORD versión 4.01, 1995-1999 MjM Software Design (McCune y Mefford 1999). Se incluyeron en la matriz de las especies sólo aquellas que presentaron un valor de importancia $\geq 5 \%$ por parcela.

\section{RESULTADOS}

Distribución y hábitat de Nectandra rudis: el registro de $N$. rudis en la Sierra de Manantlán amplía la distribución conocida de la especie y representa el primer registro para el occidente de México y el más septentrional del taxon, ya que las poblaciones conocidas de más al norte eran las del estado de Chiapas.

Se trata de una especie rara en el occidente de México, y sólo se conocen colectas de la Estación Científica Las Joyas y las cañadas húmedas que bajan hacia Casimiro Castillo y la comunidad indígena de Cuzalapa, en altitudes donde la incidencia de neblina es frecuente a lo largo del año, por lo que es de suponerse que una distribución tan restringida y una alteración de su hábitat la hace potencialmente frágil a la extinción en esta región del país.

$N$. rudis, en Chiapas, Guatemala y El Salvador, se registra entre los 1600 y 2500 $\mathrm{m}$ de altitud, en bosque mesófilo de montaña, hábitat que coincide con el que se le conoce para la Sierra de Manantlán, ya que se le ha encontrado en el mismo tipo de vegetación y en una altitud entre 1550 y $1900 \mathrm{~m}$.

La distribución altitudinal tan restringida de $N$. rudis, en la Sierra de Manantlán, es un indicador de que la especie debe tener requerimientos ambientales muy particulares que no se presentan en cualquier área. Las densidades registradas son muy bajas y su área basal representa menos del $9 \%$, lo cual es una señal de que la especie tiene poca capacidad de establecimiento y los pocos individuos que lo hacen llegan a alcanzar grandes dimensiones diamétricas y de altura.

Estructura poblacional y especies asociadas: $N$. rudis, sólo se encuentra en siete, de las 56 parcelas muestreadas, una parcela a los 1 $750 \mathrm{~m}$ de altitud, cuatro a los $1650 \mathrm{~m}$, y dos a los $1550 \mathrm{~m}$. Se registran 44 individuos, lo que representa el $4.5 \%$ de los 959 individuos consignados en 0.7 ha. La población de $N$. rudis manifiesta una altura promedio de $10 \mathrm{~m} \pm 6.60$ m. El $65 \%$ de los individuos tiene un solo tallo, el $21 \%$ dos tallos y sólo el $14 \%$ presenta más de dos tallos por debajo del $1.30 \mathrm{~m}$ de altura. De acuerdo con su densidad, la especie tiene su mayor expresión ecológica en la franja altitudinal de $1650 \mathrm{~m}$. El área basal de $N$. rudis 
representa el $8.7 \%$ del área total de las siete parcelas (Cuadro 1).

La población de $N$. rudis se estructura en cinco categorías diamétricas (Fig. 1), la cual se caracteriza por una mayor proporción de individuos en las jerarquías diamétricas pequeñas, lo que genera una gráfica en forma de "J" invertida, que indica tasas altas de establecimiento de individuos pequeños, de los cuales un número reducido alcanza tallas diamétricas mayores.

$N$. rudis presenta los mayores valores de importancia en las parcelas nueve, $11 \mathrm{y}$ 12, a los $1650 \mathrm{~m}$ de altitud; coexiste, con Cedrela odorata L., Parathesis villosa Lundell, Solanum nigricans M. Martens y Galeotti, Juglans olanchana Standley y L.O. Williams, Boehmeria ulmifolia Wedd., Prunus sp., y Prunus cortapico Kerber ex Koehne, las cuales presentan sus mayores atributos estructurales a la misma altitud (Fig. 2).

Estructura de la comunidad: en las comunidades donde se localiza $N$. rudis, en la Sierra de Manantlán, cerca del $70 \%$ de los individuos de todas las especies tienen alturas entre los 2 y $10 \mathrm{~m}$, pero hay algunos árboles que superan los $40 \mathrm{~m}$. En el estrato de arbustos y árboles pequeños se registran a Urera verrucosa (Liebm.) V.W. Steinm., R. manantlanensis Lorence, Euonymus acuminatus Benth.

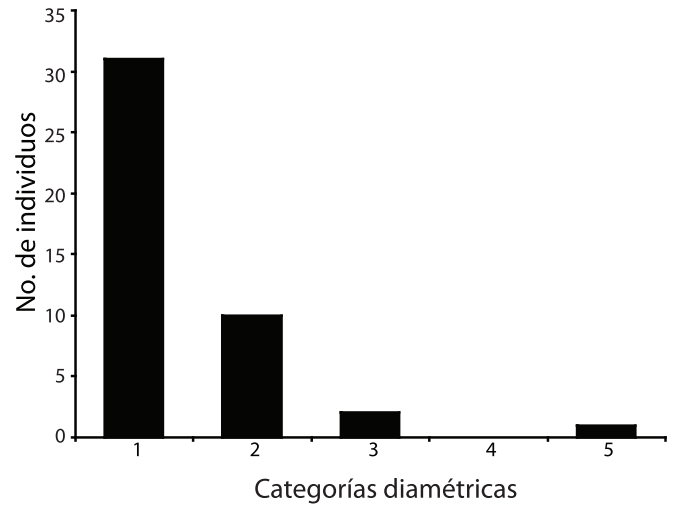

Fig. 1. Distribución diamétrica de la población de Nectandra rudis. $1=2.5-22.1 \mathrm{~cm} ; 2=22.2-41.8 \mathrm{~cm} ; 3=41.9-61.5 \mathrm{~cm}$; $4=61.6-81.2 \mathrm{~cm}$ y $5=81.3-100.9 \mathrm{~cm}$.

Fig. 1. Diameter distribution for a population of Nectandra rudis. $1=2.5-22.1 \mathrm{~cm} ; 2=22.2-41.8 \mathrm{~cm} ; 3=41.9-61.5 \mathrm{~cm}$; $4=61.6-81.2 \mathrm{~cm}$ y $5=81.3-100.9 \mathrm{~cm}$.

y Lycianthes manantlanensis A. Rodríguez y O. Vargas. Árboles de tamaño medio están representados por Clusia salvinii Donn. Sm., Gymnanthes riparia (Schldl.) Klotzsch, Meliosma nesites J.M. Johnst., Myrcianthes fragrans (Sw.) McVaugh y Robinsonella speciosa Fryx. Los árboles que presentan alturas de más de $20 \mathrm{~m}$ son Beilschmiedia manantlanensis Cuevas y Cochrane, Calatola laevigata Standley, Fraxinus uhdei (Wenz.) Lingelsh.,

CUADRO 1

Atributos estructurales de N. rudis y los encontrados en las parcelas donde esta se desarrolla

$\begin{array}{lccccccccc}\text { Um } & \text { Alt. } & \text { Ab } & \text { D } & \text { F } & \text { S } & \begin{array}{c}\text { Áb de } N . \\ \text { rudis }\end{array} & \begin{array}{c}\text { D de } N . \\ \text { rudis }\end{array} & \begin{array}{c}\text { F de } N . \\ \text { rudis }\end{array} & \begin{array}{c}\text { VI de } N \text {. } \\ \text { rudis }\end{array} \\ \text { P6 } & 1750 & 4.0349 & 98 & 67 & 34 & 0.0061 & 1 & 1 & 0.888 \\ \text { P9 } & 1650 & 1.8568 & 171 & 75 & 30 & 0.7793 & 19 & 6 & 20.360 \\ \text { P10 } & 1650 & 2.429 & 155 & 70 & 31 & 0.1717 & 4 & 4 & 1.235 \\ \text { P11 } & 1650 & 2.159 & 162 & 90 & 39 & 0.1121 & 8 & 3 & 4.488 \\ \text { P12 } & 1650 & 6.3601 & 135 & 79 & 33 & 0.8041 & 5 & 3 & 6.715 \\ \text { P13 } & 1550 & 2.4435 & 77 & 51 & 27 & 0.0862 & 3 & 2 & 3.782 \\ \text { P14 } & 1550 & 6.5705 & 155 & 89 & 35 & 0.3022 & 4 & 3 & 3.517\end{array}$

$\mathrm{Um}=$ unidad de muestreo, Alt. = altitud, $\mathrm{Ab}=$ área basal expresada en $\mathrm{m}^{2} 0.1 \mathrm{ha}^{-1}, \mathrm{D}=$ densidad expresada en número de ind.0.1 ha ${ }^{-1}, \mathrm{~F}=$ frecuencia, $\mathrm{S}=$ riqueza de especies, $\mathrm{VI}=$ valor de importancia. 


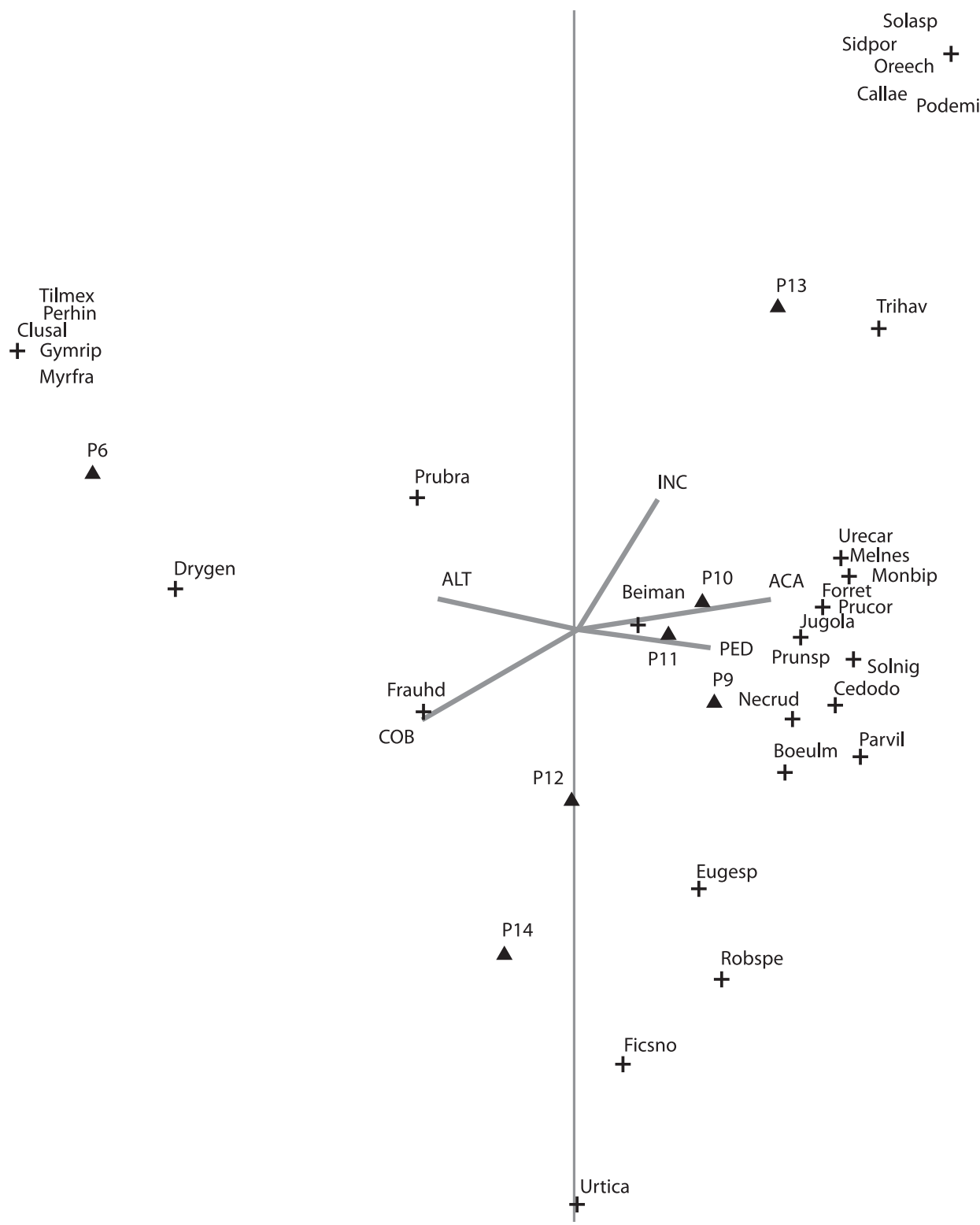

Fig. 2. Ordenación con Análisis de Correspondencia Canónica con la matriz de las especies y las variables ambientales. ALT $=$ altitud INC $=$ incendios; $\mathrm{ACA}=$ árboles caídos $; \mathrm{COB}=$ cobertura; $\mathrm{PED}=$ pedregosidad. $\mathrm{P} 1, \mathrm{P} 2 \ldots \mathrm{P} 14=$ parcela 1 , parcela $2 \ldots$ parcela 14. Beiman $=$ Beilschmiedia manantlanensis, Boeulm $=$ Boehmeria ulmifolia, Callae $=$ Calatola laevigata, Cedodo $=$ Cedrela odorata, Clusal $=$ Clusia salvinii, Drygen $=$ Drypetes gentryi, Eugesp $=$ Eugenia sp., Ficsno $=$ Ficus sp. nov., Forret $=$ Forestiera reticulata, Frauhd $=$ Fraxinus uhdei, Jugola $=$ Juglans olanchana, Melnes $=$ Meliosma nesites, Monbip = Montanoa bipinnatifida, Myrfra = Myrcianthes fragrans, Necrud = Nectandra rudis, Oreech $=$ Oreopanax echinops, Parvil $=$ Parathesis villosa, Perhin $=$ Persea hintonii, Podemi $=$ Podachaenium eminens, Prubra $=$ Prunus brachybotrya, Prucor $=$ Prunus cortapico, Prunsp $=$ Prunus sp., Robspe $=$ Robinsonella speciosa, Sidpor $=$ Sideroxylon portoricense, Solnig $=$ Solanum nigricans, Solasp $=$ Solanum $\mathrm{sp} .$, Tilmex $=$ Tilia americana var. mexicana, Trihav $=$ Trichilia havanensis, Urecar $=$ Urera verrucosa, Urtica $=$ Urticaceae.

Fig. 2. Canonical Correspondence Analysis ordination, with the matrix of species and environmental variables. ALT $=$ altitude; $\mathrm{INC}=$ fires; $\mathrm{ACA}=$ rooted trees $\mathrm{COB}=$ cover. 
Nectandra rudis, Prunus brachybotrya Zucc., Sideroxylon portoricense Urban, y Prunus cortapico. Varias de estas últimas especies junto con Ficus sp., alcanzan alturas mayores a $40 \mathrm{~m}$, comportándose como emergentes. Los bejucos están bien representados y algunos de ellos alcanzan longitudes de más de $40 \mathrm{~m}$ como Canavallia hirsutissima Sauer, Celastrus pringlei Rose, Clematix rhodocarpa Rose, Gouania lupuloides (L.) Urban, Thenardia floribunda Humb., Bonpl. y Kunth y Vitis blancoi Munson.

La riqueza varía de 27 a 39 especies y los mayores valores se registran para las parcelas 11 y 14 (Cuadro 1). La mayor densidad y área basal se encuentra en las parcelas nueve y 11 y 14 y 12, respectivamente. Los mayores valores de importancia en la comunidad son para Urera verrucosa, Nectandra rudis, Ficus sp., Beilschmiedia manantlanensis y Drypetes gentryi Monach. (Cuadro 2).
Asociación de Nectandra rudis con variables del medio: el Análisis de Correspondencia Canónica (CCA), obtiene con la matriz de las especies y la de variables ambientales, que los tres primeros ejes explican el $64.9 \%$ del total de la varianza contenida en los datos de las especies en la comunidad. El primer eje explica el $27 \%$, el segundo $20.5 \%$ y el tercero el $17.4 \%$. El primer eje se correlaciona con la altitud $(\mathrm{r}=0.647)$ y la cobertura $(\mathrm{r}=0.730)$, con la presencia de árboles caídos $(\mathrm{r}=-0.888)$ y la pedregosidad $(\mathrm{r}=-0.609)$. El eje dos con la incidencia de incendios $(\mathrm{r}=-0.686)$.

El CCA con la matriz de las especies y la de factores edáficos advierten que la profundidad del horizonte superficial y el magnesio soluble presentan las mayores correlaciones con el eje 1 ( $\mathrm{r}=0.928$ y $\mathrm{r}=-0.820$, respectivamente); el eje dos esta correlacionado con el potasio $(\mathrm{r}=-0.707)$.

CUADRO 2

Especies con los mayores valores de importancia por parcela en la comunidad donde se registra $\mathrm{N}$. rudis

$\begin{array}{lccccccc}\text { Especie } & \mathrm{P} 6 & \mathrm{P} 9 & \mathrm{P} 10 & \mathrm{P} 11 & \mathrm{P} 12 & \mathrm{P} 13 & \mathrm{P} 14 \\ \text { Beilschmiedia manantlanensis } & 5.11 & 0.00 & 9.94 & 0.00 & 4.05 & 4.02 & 5.23 \\ \text { Boehmeria ulmifolia } & 0.00 & 6.14 & 0.00 & 4.52 & 4.37 & 0.00 & 0.00 \\ \text { Cedrela odorata } & 0.00 & 5.15 & 0.00 & 4.03 & 0.00 & 0.00 & 0.00 \\ \text { Eugenia } \text { sp. } & 0.00 & 0.00 & 0.00 & 8.82 & 0.00 & 0.00 & 7.04 \\ \text { Drypetes gentryi } & 12.47 & 0.00 & 0.00 & 0.00 & 0.00 & 0.00 & 4.86 \\ \text { Ficus sp. } & 0.00 & 0.00 & 0.00 & 0.00 & 16.48 & 0.00 & 16.37 \\ \text { Forestiera reticulata } & 0.00 & 0.00 & 5.93 & 6.58 & 0.00 & 0.00 & 0.00 \\ \text { Fraxinus uhdei } & 4.90 & 0.00 & 0.00 & 0.00 & 8.18 & 0.00 & 0.00 \\ \text { Meliosma nesites } & 0.00 & 0.00 & 10.05 & 0.00 & 0.00 & 0.00 & 0.00 \\ \text { Myrcianthes fragrans } & 9.93 & 0.00 & 0.00 & 0.00 & 0.00 & 0.00 & 0.00 \\ \text { Nectandra rudis } & 0.89 & 20.36 & 5.12 & 4.49 & 6.71 & 3.78 & 3.52 \\ \text { Parathesis villosa } & 0.00 & 7.46 & 0.00 & 0.00 & 0.00 & 0.00 & 0.00 \\ \text { Prunus brachybotrya } & 6.12 & 0.00 & 0.00 & 6.32 & 0.00 & 0.00 & 0.00 \\ \text { Robinsonella speciosa } & 0.00 & 4.84 & 0.00 & 0.00 & 0.00 & 0.00 & 4.69 \\ \text { Sideroxylon portoricense } & 0.00 & 0.00 & 0.00 & 0.00 & 0.00 & 7.68 & 0.00 \\ \text { Trichilia havanensis } & 0.00 & 0.00 & 0.00 & 4.59 & 0.00 & 5.23 & 0.00 \\ \text { Urera verrucosa } & 0.00 & 15.52 & 17.60 & 13.02 & 9.50 & 15.98 & 0.00 \\ \text { Urticaceae } & 0.00 & 0.00 & 0.00 & 0.00 & 0.00 & 0.00 & 11.28\end{array}$


$N$. rudis presenta su mayor correlación con el eje $1(r=-0.440)$, por lo que es de esperarse que cualquier elemento del medio que este correlacionado con este eje, es un factor que influye en la distribución de la especie. Las variables que presentan las correlaciones más altas con éste eje son la presencia de árboles caídos, la cobertura, la profundidad del horizonte superficial y el magnesio soluble, lo que sugiere que éstos son los factores más importantes asociados con la distribución de la especie (Fig. 2 y 3 ).

\section{DISCUSIÓN}

$N$. rudis, es una especie muy estrechamente relacionada con $N$. lundellii C.K. Allen, de la cual se diferencia por tener las hojas revolutas en la base, tépalos, anteras y frutos más grandes, además de que se le ha encontrado únicamente en bosque mesófilo de montaña en altitudes por arriba de los 1550 metros, mientras que la segunda especie se le conoce del bosque tropical perennifolio y de altitudes por debajo de los $500 \mathrm{~m}$ (Rohwer 1993, Lorea 2002). El fenómeno de que las especies relacionadas del género Nectandra tienden a evitar la competencia y buscan ocupar diferentes nichos ya ha sido señalado por Rohwer (1993).

La disyunción biogeográfica que presenta $N$. rudis, es un patrón que ya ha sido registrado para otras especies que también tienen como hábitat el bosque mesófilo de montaña o ecotono entre esta comunidad vegetal y el bosque tropical subcaducifolio como Calatola laevigata Standley (Vera 1999), Capparis quirrigensis Standley (Vázquez et al. 1995), Acer skutchii Rehder (Jardel et al. 1996) y, recientemente, Desmopsis trunciflora (Schldl. y Cham.) G.E. Schatz, (Cuevas et al. 2002). Este fenómeno se ha explicado como el resultado de eventos de vicarianza, que dieron lugar a la fragmentación de un tipo de vegetación análogo al actual bosque mesófilo de montaña que presentaba una distribución más amplia en el terciario (Rzedowski y McVaugh 1966, Axelrod 1975, Rzedowski y Palacios 1977, Wolfe y
Tanai 1987). Cierto grado de incertidumbre se produce al cuestionar si la distribución que presenta $N$. rudis no es en realidad un efecto de intensidad de recolecta en los Estados intermedios entre la Sierra de Manantlán y Chiapas, incertidumbre que disminuye si se considera que se cuenta con un trabajo monográfico para el género en el Neotrópico (Rohwer 1993), y recientemente se hizo un estudio de la familia Lauraceae en el sur de México, y en ambos se restringe la distribución de $N$. rudis a los bosques mesófilos de Chiapas (Lorea 2002) y Guatemala y El Salvador (Rohwer 1993).

$\mathrm{Su}$ estructura diámetrica, en forma de "J" invertida (Fig. 2), sugiere un típico comportamiento de miembros de la familia Lauraceae, con una gran producción de semillas y plántulas, con una alta mortalidad denso-dependiente, de las cuales muy pocas alcanzan el estado adulto (Martínez 1985).

Varios son los factores ambientales que influyen en la distribución y abundancia de $N$. rudis, pero el hecho que sus mayores valores de importancia se presenten donde hay mayor cantidad de árboles caídos y menor cobertura es una respuesta observada en otras lauráceas, las cuales buscan espacios con mayor incidencia de luz que les permitan incrementar sus tasas fotosintéticas y de esta forma alcanzar el dosel (Del Amo 1985). N. rudis es una especie umbrófila que año con año forma tapices de plántulas alrededor de la planta madre, de las que pocas sobreviven, pero las que lo hacen tienen la capacidad de vivir un tiempo como umbrófilas y responder como heliófitas ante la apertura de claros por la caída de ramas o árboles (Gómez y Vázquez 1985).

$N$. rudis presenta un típico comportamiento de una especie nómada (Martínez 1985), ya que produce semillas grandes y de viabilidad corta, que germinan cuando hay altos contenidos de humedad y forman grandes concentraciones de plántulas de las cuales unas pocas sobreviven e incrementan su tamaño cuando se abre un claro.

La respuesta positiva de $N$. rudis a suelos someros y de mayor pedregosidad podría estar ligado a la probabilidad de que a menor 


$+\begin{gathered}\text { Tilmex } \\ \text { Gymrip } \\ \text { Clusal } \\ + \text { Perhin } \\ \text { Myrfra }\end{gathered}$

P6

Fig. 3. Ordenación con Análisis de Correspondencia Canónica con la matriz de las especies y las variables edáficas. PHS $=$ profundidad del horizonte superficial; $\mathrm{KN}=$ potasio; $\mathrm{MGS}=$ magnesio soluble. $\mathrm{P} 1, \mathrm{P} 2 \ldots \mathrm{P} 14=$ parcela 1 , parcela $2 \ldots$ parcela 14. Beiman $=$ Beilschmiedia manantlanensis, Boeulm $=$ Boehmeria ulmifolia, Callae $=$ Calatola laevigata, Cedodo $=$ Cedrela odorata , Clusal $=$ Clusia salvinii, Drygen $=$ Drypetes gentryi, Eugesp $=$ Eugenia sp., Ficsno $=$ Ficus sp. nov., Forret $=$ Forestiera reticulata, Frauhd $=$ Fraxinus uhdei, Gymrip $=$ Gymnathes riparia, Jugola = Juglans olanchana, Melnes $=$ Meliosma nesites, Monbip = Montanoa bipinnatifida, Necrud = Nectandra rudis, Oreech = Oreopanax echinops, Parvil $=$ Parathesis villosa , Perhin $=$ Persea hintonii, Prubra $=$ Prunus brachybotrya, Prucor $=$ Prunus cortapico, Prunsp $=$ Prunus sp., Robspe $=$ Robinsonella speciosa, Sidpor $=$ Sideroxylon portoricense, Solnig $=$ Solanum nigricans, Solasp $=$ Solanum $\mathrm{sp} .$, Tilmex $=$ Tilia americana var. mexicana, Trihav $=$ Trichilia havanensis, Urecar $=$ Urera verrucosa, Urtica $=$ Urticaceae.

Fig. 3. Canonical Correspondence Analysis ordination, with the matrix of species and soil variables. PHS = superficial horizon depth; $\mathrm{KN}=$ potasium; $\mathrm{MGS}=$ soluble magnesium. $\mathrm{P} 1, \mathrm{P} 2 \ldots \mathrm{P} 14=$ plot 1 , plot $2 \ldots$ plot 14 
profundidad y mayor pedregosidad, los árboles están menos arraigados, lo que incrementa la posibilidad de derriba y abren claros a los cuales responden los individuos jóvenes de la especie (Del Amo 1985).

El hecho de que $N$. rudis se vea favorecida por la presencia de magnesio soluble, podría atribuirse a que este elemento es parte de la molécula de la clorofila, lo que influiría en el incremento de las tasas de fotosíntesis de las plantas de los claros, y con ello ratificar lo dicho por FitzPatrick (1996) y Ortíz y Ortíz (1980) en cuanto a que este elemento es considerado esencial para la fotosíntesis, coadyuva en la translocación del almidón y en la absorción de otros nutrimentos (Ortíz y Ortíz 1980), es importante para que la planta almacene almidón en los tejidos de reserva (Shuman 1994), además de que hay algunas evidencias de que la baja disponibilidad de este elemento podría limitar el crecimiento de las plántulas en los suelos forestales (Burslem et al. 1996).

\section{AGRADECIMIENTOS}

A la Universidad de Guadalajara por el apoyo económico otorgado a través del proyecto "Análisis de gradientes de la vegetación de cañadas en la Sierra de Manantlán". A Francisco Lorea Hernández por su amabilidad en revisar y corroborar la identificación del taxon. A los compañeros de trabajo de campo Luis Guzmán, Enrique V. Sánchez y Jorge Aragón. Miguel Olvera Vargas y Peter Gerritsen revisaron e hicieron comentarios que mejoraron el escrito.

\section{RESUMEN}

Nectandra rudis es una especie rara en el occidente de México. Analizamos la población y la comunidad donde se desarrolla, en la Sierra de Manantlan, Jalisco, México. Establecimos cuatro parcelas de $60 \times 48 \mathrm{~m}$ con diferencias altitudinales de $100 \mathrm{~m}$ entre sí. En cada una seleccionamos aleatoriamente diez círculos de $100 \mathrm{~m}^{2}$ cada uno, en los cuales medimos los diámetros normales y las alturas de las especies leñosas con diámetro $\geq 2.5 \mathrm{~cm}$. Para cada especie y por parcela determinamos el área basal, la densidad, la frecuencia, la riqueza de especies y los valores de importancia. Establecimos la estructura vertical y diamétrica de $N$. rudis. Hicimos una ordenación directa con la matriz de especies y las variables edáficas y ambientales. El registro de $N$. rudis en la Sierra de Manantlán representa el primer registro para el occidente de México y el más septentrional del taxon. La población se estructura en cinco categorías diamétricas y genera una curva en forma de "J" invertida. La riqueza de especies en las parcelas donde se encuentra $N$. rudis, varía de 27 a 39, y los mayores valores de importancia son para Urera verrucosa, $N$. rudis y Ficus sp. La ordenación directa permite postular a la presencia de árboles caídos, la cobertura, la profundidad del horizonte superficial y el magnesio soluble, como los factores ambientales de mayor influencia en la distribución y abundancia de $N$. rudis.

Palabras clave: Análisis de Correspondencia Canónica, estructura poblacional, Nectandra rudis, occidente de México, Sierra de Manantlán.

\section{REFERENCIAS}

Axelrod, D.I. 1975. Evolution and biogeography of Madrean-Tethyan sclerophyll vegetation. Ann. Mo. Bot. Gard. 62: 280-334.

Burslem, D.F.R.P., P.J. Grubb \& I.M. Turner. 1996. Responses to simulated drought and elevated nutrient supply among shade tolerant tree seedling of lowland tropical forest in Singapore. Biotropica 28: 636-648.

Cuevas, R. 2002. Análisis de gradientes de la vegetación de la cañada El Tecolote, en la Sierra de Manantlán, Jalisco, México. Tesis Doctoral, Colegio de Postgraduados, Montecillo, Estado de México, México.

Cuevas, R., L. López \& E. García. 2002. Primer registro de Desmopsis trunciflora (Schlecht. y Cham.) G.E. Schatz (Annonaceae) para el occidente de México y análisis de su población en la Sierra de Manantlán, Jalisco. Act. Bot. Mex. 58: 7-18.

Curtis, J.T. \& R.P. McIntosh. 1951. An upland forest continuum in the prairie forest border region of Wisconsin. Ecology 32: 476-496.

Del Amo, S. 1985. Algunos aspectos de la influencia de la luz sobre el crecimiento de estados juveniles de especies primarias, p. 79-92. In A. Gómez \& S. Del Amo (eds.). Investigaciones sobre la regeneración de selvas altas en Veracruz, México, II. Editorial Alambra Mexicana, Xalapa, Veracruz, México.

FitzPatrick, E.A. 1996. Introducción a la ciencia de los suelos. Editorial Trillas, México D.F, México. 
Gómez, A. \& C. Vázquez. 1985. Estudios sobre la regeneración de selvas en regiones cálido-húmedas de México, p. 1-26. In A. Gómez \& S. Del Amo (eds.). Investigaciones sobre la regeneración de selvas altas en Veracruz, México, II. Editorial Alambra Mexicana, Xalapa, Veracruz, México.

Jardel, E.J., R. Cuevas, A.L. Santiago, M.E. Muñoz \& J. Aragón. 1996. Nueva localidad y características de la población de Acer skutchii Rehder en la Sierra de Manantlán, Jalisco, México. Act. Bot. Mex. 35: 13-24.

Lorea, F. 2002. La familia Lauraceae en el sur de México: diversidad, distribución y estado de conservación. Bol. Soc. Bot. México 71: 59-70.

Martínez, M. 1985. Claros, ciclos vitales de los árboles tropicales y regeneración natural de las selvas altas perennifolias, p. 191-240. In A. Gómez \& S. Del Amo (eds.). Investigaciones sobre la regeneración de selvas altas en Veracruz, México, II. Editorial Alambra Mexicana, Xalapa, Veracruz, México.

Martínez, L.M., J.J. Sandoval \& R.D. Guevara. 1991 El clima en la Reserva de la Biosfera Sierra de Manantlán (Jalisco-Colima, México) y en su área de influencia. Agrociencia Ser. Agua-Suelo-Clima 2: $107-119$

McCune, B. \& M.J. Mefford. 1999. PC-ORD. Multivariate Analysis of Ecological Data. Version 4.01 MjM Software Design, Gleneden Beach, Oregon, EEUU.

Olvera, M., S. Moreno \& B. Figueroa. 1996. Sitios permanentes para la investigación silvícola. Universidad de Guadalajara, Guadalajara, Jalisco, México.

Orozco, H. 2001. Ecología del paisaje del ejido Barranca de La Naranjera, Jalisco. Tesis profesional, Universidad de Guadalajara, Guadalajara, Jalisco, México.

Ortíz, B. \& C.A. Ortíz. 1980. Edafología. Universidad Autónoma de Chapingo. Chapingo, Estado de México, México.

Rohwer, J.G. 1993. Lauraceae: Nectandra. Flora Neotropica 60: 1-333.
Rzedowski, J. 1978. Vegetación de México. LIMUSA, México D.F., México.

Rzedowski, J. \& R. McVaugh. 1966. La vegetación de Nueva Galicia. Contr. Univ. Michigan Herb. 9: $1-123$.

Rzedowski, J. \& R. Palacios. 1977. El bosque de Engelhardtia (Oreomunnea) mexicana en la región de la Chinantla, Oaxaca, México, una reliquia del Cenozoico. Bol. Soc. Bot. Méx. 36: 93-123.

Shuman, L.M. 1994. Mineral nutrition, p. 149-182. In R.E. Wilkinson (ed.). Plant environment interactions. Marcel Dekker, Nueva York, EEUU.

ter Braak, C.J.F. \& P. Smilauer. 1998. CANOCO reference manual and user's guide to canoco for Windows: Software for canonical community ordination (version 4). Microcomputer power. Ithaca, Nueva York, EEUU.

Vázquez, J.A., R. Cuevas, T. Cochrane, H.H. Iltis, F. J. Santana \& L. Guzmán. 1995. Flora de Manantlán. Sida Bot. Miscellany 13: 212.

Vázquez, J.A. \& T.J. Givnish. 1998. Altitudinal gradients in tropical forest composition, structure, and diversity in the Sierra de Manantlán. J. Ecol. 86: 999-1020.

Vera, P. 1999. Revisión del género Calatola (Icacinaceae) en México. Tesis de Maestría, Colegio de Postgraduados, Montecillo, Estado de México, México.

Wendt, T. 1998. Composición, afinidades florísticas y orígenes de la flora arbórea del dosel de los bosques tropicales húmedos de la vertiente mexicana del Atlántico, p. 581-664. In T.P. Ramamoorthy, R. Bye, A. Lot \& J. Fa (comp.). Diversidad biológica de México. Instituto de Biología y UNAM, México D.F., México.

Wolfe, J.A. \& T. Tanai. 1987. Systematic, phylogeny, and distribution of Acer (maples) in the Cenozoic of western North America. J. Fac. Sci. Hokkaido Univ. 22: 1-246. 